\title{
NEIGHBORHOODS AND PARTIAL SUMS OF CERTAIN MEROMORPHICALLY MULTIVALENT FUNCTIONS
}

\author{
JIN-LIN LIU
}

\begin{abstract}
By making use of the familiar concept of neighborhoods of analytic functions, the author proves an inclusion relations associated with the $(n, \delta)$-neighborhoods of a subclass $Q_{k}[p, \alpha ; A, B]$ which was introduced by Srivastava, Hossen and Aouf. The partial sums of the functions in $Q_{k}[p, \alpha ; A, B]$ are also considered.
\end{abstract}

\section{Introduction}

Let $\sum_{p, k}$ be the class of functions of the form

$$
f(z)=z^{-p}+\sum_{n=k}^{\infty} a_{n+p-1} z^{n+p-1}, \quad(p, k \in N=\{1,2,3, \ldots\}),
$$

which are analytic and $p$-valent in the punctured unit disk

$$
U^{*}=\{z: z \in C \text { and } 0<|z|<1\}=U \backslash\{0\} .
$$

In recent years, many important properties and characteristics of various interesting sbuclasses of the class $\sum_{p, k}$ of meromorphically $p$-valent functions were investigated extensively by Srivastava et al. 7], Owa et al. [5], Yang [9], Liu and Srivastava [3, 4] and other $([2,8])$. In [7] , Srivastava, Hossen and Aouf introduced and studied a subclass $Q_{k}[p, \alpha ; A, B]$ of $\sum_{p, k}$ as following.

Definition. For fixed parameters $A$ and $B$, with $-1 \leq A<B \leq 1, A+B \geq 0$, and $0<B \leq 1$. A function $f \in \sum_{p, k}$ is said to be in the class $Q_{k}[p, \alpha ; A, B]$ of meromorphically $p$-valent functions in $U$ if and only if

$$
\left|\frac{\left(z f^{\prime}(z) / f(z)\right)+p}{B\left(z f^{\prime}(z) / f(z)+[p B+(A-B)(p-\alpha)]\right.}\right|<1 \quad\left(z \in U^{*} ; \quad 0 \leq \alpha<p\right) .
$$

Many interesting properties of the class $Q_{k}[p, \alpha ; A, B]$ were obtained by Srivastava, Hossen and Aouf [].

Received February 09, 2006.

2000 Mathematics Subject Classification. 30C45.

Key words and phrases. Meromorphic function, $p$-valent function, neighborhoods, partial sums. 
In the present paper, we shall discuss the properties of Neighborhoods and partial sums of the subclass $Q_{k}[p, \alpha ; A, B]$

\section{Main results}

Following the earlier works (based upon the familiar concept of neighborhoods of analytic functions) by Goodman [1] and Ruscheweyh [6], we begin by introducing here the $\delta$-neighborhood of a function $f \in \sum_{p, k}$ of the from (1.1) by means of the definition

$$
\begin{aligned}
N_{\delta}(f)= & \left\{g \in \sum_{p}: g(z)=z^{-p}+\sum_{n=k}^{\infty} b_{n+p-1} z^{n+p-1}\right. \text { and } \\
& \sum_{n=k}^{\infty} \frac{(1+B)(n+2 p-1)+(p-\alpha)(B-A)}{(p-\alpha)(B-A)}\left|a_{n+p-1}-b_{n+p-1}\right| \leq \delta \\
& \quad(-1 \leq A<B \leq 1 ; \quad \delta \geq 0)\}
\end{aligned}
$$

Making use of definition (2.1), we now prove the following result.

Theorem 1. Let $f \in Q_{k}[p, \alpha ; A, B]$ be given by (1.1). If $f$ satisfies the inclusing condition

$$
\left(f(z)+\varepsilon z^{-p}\right)(1+\varepsilon)^{-1} \in Q_{k}[p, \alpha ; A, B] \quad(\varepsilon \in C ;|\varepsilon|<\delta ; \delta>0),
$$

then

$$
N_{\delta}(f) \subset Q_{k}[p, \alpha ; A, B]
$$

Proof. It is easily seen from (1.2) that a function $f \in Q_{k}[p, \alpha ; A, B]$ if and only if

$$
\frac{z f^{\prime}(z)+p f(z)}{B z f^{\prime}(z)+[p B+(A-B)(p-\alpha)] f(z)} \neq \sigma \quad(z \in U ; \sigma \in C,|\sigma|=1)
$$

which is equivalent to

$$
\frac{(f \times h)(z)}{z^{-p}} \neq 0 \quad(z \in U)
$$

where, for convenience

$$
\begin{aligned}
h(z) & =z^{-p}+\sum_{n=k}^{\infty} \frac{(B \sigma-1)(n+2 p-1)+\sigma(p-\alpha)(A-B)}{\sigma(p-\alpha)(A-B)} z^{n+p-1} \\
& =z^{-p}+\sum_{n=k}^{\infty} c_{n+p-1} z^{n+p-1} .
\end{aligned}
$$


We easily find from (2.6) that

$$
\begin{aligned}
\left|c_{n+p-1}\right| & =\left|\frac{(B \sigma-1)(n+2 p-1)+\sigma(p-\alpha)(A-B)}{\sigma(p-\alpha)(A-B)}\right| \\
& \leq \frac{(1+B)(n+2 p-1)+(p-\alpha)(B-A)}{(p-\alpha)(B-A)} .
\end{aligned}
$$

Furthermore, under the hypothesis of the theorem, (2.5) yields the following inequality

$$
\left|\frac{(f \times h)(z)}{z^{-p}}\right| \geq \delta \quad(z \in U ; \delta>0) .
$$

Now, we let

$$
\varphi(z)=z^{-p}+\sum_{n=k}^{\infty} b_{n+p-1} z^{n+p-1} \in N_{\delta}(f)
$$

then

$$
\begin{aligned}
& \left|\frac{[f(z)-\varphi(z)] \times h(z)}{z^{-p}}\right| \\
& \quad=\left|\sum_{n=k}^{\infty}\left(a_{n+p-1}-b_{n+p-1}\right) c_{n+p-1} z^{n+2 p-1}\right| \\
& \quad \leq \sum_{n=k}^{\infty} \frac{(1+B)(n+2 p-1)+(p-\alpha)(B-A)}{(p-\alpha)(B-A)}\left|a_{n+p-1}-b_{n+p-1}\right| \cdot\left|z^{n+2 p-1}\right| \\
& \quad<\delta \quad(z \in U ; \delta>0) .
\end{aligned}
$$

Thus, for any complex number $\sigma$ such that $|\sigma|=1$, we have

$$
\frac{(\varphi \times h)(z)}{z^{-p}} \neq 0 \quad(z \in U),
$$

which implies that $\varphi \in Q_{k}[p, \alpha ; A, B]$. The proof of the theorem is thus completed.

Next, we prove

Theorem 2. Let $f \in \sum_{p, k}$ be given by (1.1) and define the partial sums $s_{m}(z)$ by

$$
s_{m}(z)= \begin{cases}z^{-p} & m=1,2, \ldots k-1 \\ z^{-p}+\sum_{n=k}^{m} a_{n+p-1} z^{n+p-1} & m=k, k+1, \ldots\end{cases}
$$

Suppose also that

$$
\begin{aligned}
& \sum_{n=k}^{\infty} l_{n+p-1}\left|a_{n+p-1}\right| \leq 1 \\
(\text { where } & \left.l_{n+p-1}=\frac{(1+B)(n+2 p-1)+(p-\alpha)(B-A)}{(p-\alpha)(B-A)}\right) .
\end{aligned}
$$

Then 
(i) $f \in Q_{k}[p, \alpha ; A, B]$;

(ii)

$$
\begin{aligned}
& \operatorname{Re}\left(\frac{f(z)}{s_{m}(z)}\right)>1-\frac{1}{l_{m+p}} \quad(z \in U ; m=k, k+1, \ldots) \\
& \text { and }\left(\frac{s_{m}(z)}{f(z)}\right)>\frac{l_{m+p}}{1+l_{m+p}} \quad(z \in U ; m=k, k+1, \ldots) .
\end{aligned}
$$

Each of the bounds in (2.12) and (2.13) is the best possible.

Proof. (i) It is not difficult to see that

$$
z^{-p} \in Q_{k}[p, \alpha ; A, B] .
$$

Thus, from Theorem 1 and hypothesis (2.11) of Theorem 2, we have

$$
N_{1}\left(z^{-p}\right) \subset Q_{k}[p, \alpha ; A, B],
$$

which shows that $f \in Q_{k}[p, \alpha ; A, B]$.

(ii) Under the hypothesis in part (ii) of Theorem 2, we can see from (2.11) that

$$
l_{n+p}>l_{n+p-1}>1 \quad(n=k, k+1, \ldots) .
$$

Therefore, we have

$$
\sum_{n=k}^{m}\left|a_{n+p-1}\right|+l_{m+p} \sum_{n=m+1}^{\infty}\left|a_{n+p-1}\right| \leq \sum_{n=k}^{\infty} l_{n+p-1}\left|a_{n+p-1}\right|
$$

by using hypothesis (2.11) of Theorem 2 again.

Upon setting

$$
\begin{aligned}
g_{1}(z) & =l_{m+p}\left[\frac{f(z)}{s_{m}(z)}-\left(1-\frac{1}{l_{m+p}}\right)\right] \\
& =1+\frac{l_{m+p} \sum_{n=m+1}^{\infty} a_{n+p-1} z^{n+2 p-1}}{1+\sum_{n=k}^{m} a_{n+p-1} z^{n+2 p-1}} \quad(m \geq k),
\end{aligned}
$$

and applying (2.14), we find that

$$
\begin{aligned}
\left|\frac{g_{1}(z)-1}{g_{1}(z)+1}\right| & \leq \frac{l_{m+p} \sum_{n=m+1}^{\infty}\left|a_{n+p-1}\right|}{2-2 \sum_{n=k}^{m}\left|a_{n+p-1}\right|-l_{m+p} \sum_{n=m+1}^{\infty}\left|a_{n+p-1}\right|} \\
& \leq 1 \quad(z \in U, m \geq k),
\end{aligned}
$$


which readily yields inequality (2.12).

If we take

$$
f(z)=z^{-p}-\frac{z^{m+2 p-1}}{l_{m+p}} \quad(m \geq k),
$$

then

$$
\frac{f(z)}{s_{m}(z)}=1-\frac{z^{m+3 p-1}}{l_{m+p}} \rightarrow 1-\frac{1}{l_{m+p}} \quad(z \rightarrow 1-),
$$

which shows that the bound in (2.12) is the best possible.

Similarly, if we put

$$
\begin{aligned}
g_{2}(z) & =\left(1+l_{m+p}\right)\left(\frac{s_{m}(z)}{f(z)}-\frac{l_{m+p}}{1+l_{m+p}}\right) \\
& =1-\frac{\left(1+l_{m+p}\right) \sum_{n=m+1}^{\infty} a_{n+p-1} z^{n+2 p-1}}{1+\sum_{n=k}^{\infty} a_{n+p-1} z^{n+2 p-1}} \quad(m \geq k),
\end{aligned}
$$

and make use of (2.14), we can deduce that

$$
\begin{aligned}
\left|\frac{g_{2}(z)-1}{g_{2}(z)+1}\right| & \leq \frac{\left(1+l_{m+p}\right) \sum_{n=m+1}^{\infty}\left|a_{n+p-1}\right|}{2-2 \sum_{n=k}^{m}\left|a_{n+p-1}\right|-\left(l_{m+p}-1\right) \sum_{n=m+1}^{\infty}\left|a_{n+p-1}\right|} \\
& \leq 1 \quad(z \in U, m \geq k),
\end{aligned}
$$

which leads us immediately to assertion (2.13) of the theorem.

The bound in (2.13) is sharp with the extremal function given by (2.17). The proof of Theorem 2 is completed.

\section{Acknowledgement}

The preset investigation is supported by Jiangsu Gaoxiao Natural Science Foundation (04KJB110154).

\section{References}

[1] A. W. Goodman, Univalent functions and nonanalytic curves, Proc. Amer. Math. Soc. 8(1957), 598-601.

[2] S. B. Joshi and H. M. Srivastava, A certain family of meromorphically multivalent functions, Computers Math. Applic. 38(1999), 201-211. 
[3] J.-L. Liu and H. M. Srivastava, A linear operator and associated families of meromorphically multivalent functions, J. Math. Anal. Appl. (2001), 566-581.

[4] J.-L. Liu and H. M. Srivastava, Classes of meromorphically multivalent functions associated with the generalized hypergeometric function, Math. Comput. Modelling 39(2004), 21-34.

[5] S. Owa, H. E. Darwish and M. K. Aouf, Meromorphically multivalent functions with positive and fixed second coefficients, Math. Japon. 46(1997), 231-236.

[6] S. Ruscheweyh, Neighborhoods of univalent functions, Proc. Amer. Math. Soc. 81(1981), $521-527$.

[7] H. M. Srivastava, H. M. Hossen and M. K. Aouf, A unified presentation of some classes of meromorphically multivalent functions, Computers Math. Applic. 38(1999), 63-70.

[8] H. M. Srivastava and S. Owa (Editors), Curent Topics in Analytic Function Theory, World Scientific, Singapore, 1992.

[9] D.-G. Yang, Subclasses of multivalent p-valent convex functions (in Chinese), J. Math. Res. Exposition 20(2000), 215-219.

Department of Mathematics, Yangzhou University, Yangzhou 225002, Jiangsu, People's Republic of China.

E-mail: jlliu@yzu.edu.cn 